\title{
13.3
}

\section{Хаотический потенциал заряженных дислокаций в гетероконтактах III-нитридов}

\author{
(C) В.Б. Бондаренко ${ }^{1}$, А.В. Филимонов ${ }^{1,2, \uparrow}$, Ravi Kumar $^{3}$ \\ ${ }^{1}$ Санкт-Петербургский политехнический университет Петра Великого, Санкт-Петербург, Россия \\ ${ }^{2}$ Санкт-Петербургский национальный исследовательский Академический университет им. Ж.И. Алфёрова РАН, \\ Санкт-Петербург, Россия \\ ${ }^{3}$ Indian Institute of Technology Madras, Chennai, India \\ ๑E-mail: filimonov@rphf.spbstu.ru
}

Поступило в Редакцию 28 августа 2020 г.

В окончательной редакции 15 сентября 2020 г.

Принято к публикации 16 сентября 2020 г.

Исследуется структура хаотического потенциала в гетероконтактах III-нитридов, обусловленного электростатическим полем заряженных дислокаций. С учетом пространственной дисперсии диэлектрического отклика двумерного электронного газа определены амплитуда и масштаб хаотического потенциала в плоскости контакта. Показана зависимость параметров хаотического потенциала от плотности поверхностных состояний и концентрации дислокаций.

Ключевые слова: хаотический потенциал, естественный размерный эффект, гетероконтакт III-нитридов, двумерный электронный газ.

DOI: 10.21883/PJTF.2021.01.50450.18532

Одной из возможных причин снижения подвижности носителей в двумерной электронной подсистеме гетероконтактов на основе III-нитридов является рассеяние носителей на хаотическом потенциале заряженных дислокаций [1-3]. Очевидно, что интенсивность этого процесса зависит от плотности данных протяженных дефектов в гетероконтакте и распределения электронного заряда на дислокационных состояниях.

Формальное описание приконтактной подсистемы в рамках одномерной модели изгиба зон может оказаться не вполне корректным из-за наличия естественного размерного эффекта в области пространственного заряда полупроводника [4]. Отсутствие усреднения поля по большому количеству зарядов означает наличие флуктуаций напряженности поля и хаотического потенциала в плоскости локализации двумерного электронного газа. Кроме того, взаимодействие, обусловленное неоднородным электрическим полем системы линейно распределенных зарядов, фактически носит самосогласованный характер, поскольку конечны по величине как плотность поверхностных состояний, так и плотность состояний на дислокациях. С учетом известных особенностей двумерных электронных систем [5], связанных с изменениями их проводимости в зависимости от степени беспорядка, представляется актуальным исследование структуры хаотического потенциала заряженных дислокаций в гетероконтактах полупроводниковых нитридных соединений. Цель настоящей работы - оценить параметры хаотического потенциала заряженных линейных дефектов в контактах III-нитридов.

Для определенности рассмотрим гетероконтакт $\mathrm{AlGaN} / \mathrm{GaN}$, в котором прорастающие дислокации несоответствия с поверхностной концентрацией $N_{d i s l}$ будем представлять в виде нормально ориентированных к плоскости контакта линейных дефектов. При некоррелированной локализации указанных протяженных дефектов их распределение по количеству является пуассоновским. Таким образом, вероятность обнаружить $N$ дислокаций на участке контакта площадью $S=\pi R^{2}$ равна

$$
p(N)=\frac{\langle N\rangle^{N} \exp (-\langle N\rangle)}{N !},
$$

где $\langle N\rangle=N_{d i s l} S-$ среднее количество этих дефектов. Ввиду возникающих механических напряжений в контакте полярных кристаллов $\mathrm{AlN}$ и $\mathrm{GaN}$ возникает значительный пьезоэффект, обусловливающий изгиб зон $\mathrm{GaN}$ более половины ширины запрещенной зоны $\left(E_{g}=3.4 \mathrm{eV}\right)$. Как правило, канальный слой формируется на основе нелегированного GaN. В этом случае объемный заряд в приконтактной области изгиба зон формируется главным образом отрицательно заряженными дислокациями. В приближении больших изгибов зон данные протяженные дефекты в пределах области пространственного заряда можно полагать равномерно заряженными с некоторой линейной плотностью $\lambda$. Без потери общности можно считать, что соответствующие линейные дефекты в основном находятся только в одном полупроводнике. Если в гетероконтакте имеется делокализованный поверхностный заряд, то при высокой плотности поверхностных состояний $D_{s}$ (порядка $10^{14} \mathrm{~cm}^{-2} \cdot \mathrm{eV}^{-1}$ и более) для определения параметров хаотического поля имеется возможность воспользоваться методом электростатических изображений [6]. При этом рассматриваемая система аналогична совокупности диполей с линейно распределенным зарядом в их 
плечах. Поле произвольно выбранной дислокации будем определять в полярной системе координат, в которой $\rho$ - радиальная координата, определяющая расстояние от дислокации в плоскости контакта. Интегрирование вдоль заряженной дислокации в пределах области пространственного заряда шириной $L_{0}$ дает величину напряженности поля в плоскости контакта

$$
F_{i}(\rho)=\frac{2 \lambda}{\varepsilon}\left(\frac{1}{\rho}-\frac{1}{\sqrt{\rho^{2}+L_{0}^{2}}}\right) .
$$

Здесь $\varepsilon-$ диэлектрическая проницаемость среды, в которой находится $i$-я дислокация. При изгибах зон в гетероконтакте, во много раз превышающих характерную тепловую энергию носителей, вполне применимо приближение полностью обедненного слоя, в рамках которого ширина области пространственного заряда имеет вид

$$
L_{0}=\sqrt{\frac{\varepsilon U_{0}}{2 \pi e \lambda N_{d i s l}}},
$$

где $U_{0}-$ величина изгиба зон. Статистический анализ возникающих неоднородностей электрического поля [4] приводит к соответствующей амплитуде

$$
\delta F=\frac{4 \lambda \sqrt{\pi N_{d i s l}}}{\varepsilon} .
$$

При указанных значениях плотности поверхностных состояний возможна непосредственная оценка величины амплитуды хаотического потенциала в плоскости локализации двумерного электронного газа. В этих условиях величина неоднородностей потенциала может быть определена в форме, известной как приближение Томаса-Ферми, в рамках которого флуктуации плотности заряда должны следовать за изменениями потенциала:

$$
\delta \sigma=e D_{s} \delta U
$$

Разумеется, здесь предполагается малость возмущения потенциала по сравнению с энергией Ферми электрона в поверхностной зоне и пренебрегается изменением плотности состояний. Учитывая теперь линейную зависимость напряженности поля от поверхностного заряда $F=4 \pi \sigma / \varepsilon$ (см. (4) и (5)), можно связать величину $\delta U$ с параметрами системы:

$$
\delta U=\frac{\lambda}{e D_{s}} \sqrt{\frac{N_{d i s l}}{\pi}} .
$$

В случае широкого диапазона значений плотности электронных состояний в гетероконтакте следует провести более детальный анализ возникающих флуктуаций потенциала с использованием функции диэлектрического отклика поверхностной подсистемы [7]. C учетом преобразования по Фурье-Бесселю для потенциальной энергии электрона в поле заряженной дислокации в пространстве волновых векторов $q$ имеем

$$
V_{i}(q)=\frac{e \lambda}{q^{2}}\left[1-\exp \left(-q L_{0}\right)\right] .
$$

Экранированный потенциал, созданный $i$-й дислокацией в плоскости контакта, определяется стандартным методом:

$$
U_{i}(\rho)=\int_{0}^{\infty} U_{i}(q) J_{0}(\rho q) q d q,
$$

где $J_{0}(s)$ - функция Бесселя первого рода нулевого порядка, $U_{i}(q)=V_{i}(q) / \varepsilon(q)$ - образ Фурье-Бесселя экранированного потенциала, $\varepsilon(q)-$ функция диэлектрического отклика среды. Согласно теории экранирования, в сильно вырожденных двумерных электронных системах соответствующая диэлектрическая проницаемость имеет вид [8]:

$$
\varepsilon(q)=\frac{\varepsilon_{1}+\varepsilon_{2}}{2}\left(1+\frac{q_{s}}{q}\right) .
$$

Здесь $\varepsilon_{1}$ и $\varepsilon_{2}$ - диэлектрические проницаемости контактирующих полупроводников (для $\mathrm{AlGaN} / \mathrm{GaN}$ и других гетероконтактов III-нитридов $\varepsilon_{1} \approx \varepsilon_{2} \sim 10$ [9]), $q_{s}=4 \pi e^{2} D_{s} /\left(\varepsilon_{1}+\varepsilon_{2}\right)$ - параметр экранирования в двумерной электронной системе. Усреднение получаемого потенциала по площадке радиуса $R$ в плоскости поверхности контакта приводит к значению величины вклада одной заряженной дислокации $\left\langle U_{i}\right\rangle(R)$. После изменения порядка интегрирования в выражении для $\left\langle U_{i}\right\rangle(R)$ и учета среднеквадратичного отклонения в распределении (1) можно определить величины флуктуации потенциальной энергии электрона с характерным масштабом $R$ :

$$
\delta U(R)=\frac{4 e \lambda \sqrt{\pi N_{d i s l}}}{\varepsilon_{1}+\varepsilon_{2}} \int_{0}^{\infty} \frac{1-\exp \left(-q L_{0}\right)}{q\left(q+q_{s}\right)} J_{1}(q R) d q .
$$

Здесь $J_{1}(s)$ - функция Бесселя первого рода первого порядка. Исследование интеграла, входящего в (10), показывает, что при типичном условии $q_{s} L_{0} \gg 1$ максимальное значение $\delta U(R)$ достигается при некотором размере площадки $R=R_{0}$ :

$$
R_{0} \approx \sqrt{\frac{2 L_{0}}{q_{s}}}
$$

По смыслу радиус $R_{0}$ задает порядок величины размера области неоднородности потенциала в гетероконтакте. Вычисление $\delta U$ можно провести непосредственной подстановкой выражения (11) в формулу (10):

$$
\delta U=\frac{4 e \lambda L_{0} \sqrt{\pi N_{\text {disl }}}}{\varepsilon_{1}+\varepsilon_{2}} \Phi\left(q_{s} L_{0}\right) .
$$

Здесь введенная функция $\Phi(y)$ имеет вид

$$
\Phi(y)=\int_{0}^{\infty} \frac{1-\exp (-x)}{x(x+y)} J_{1}(x \sqrt{2 / y}) d x .
$$




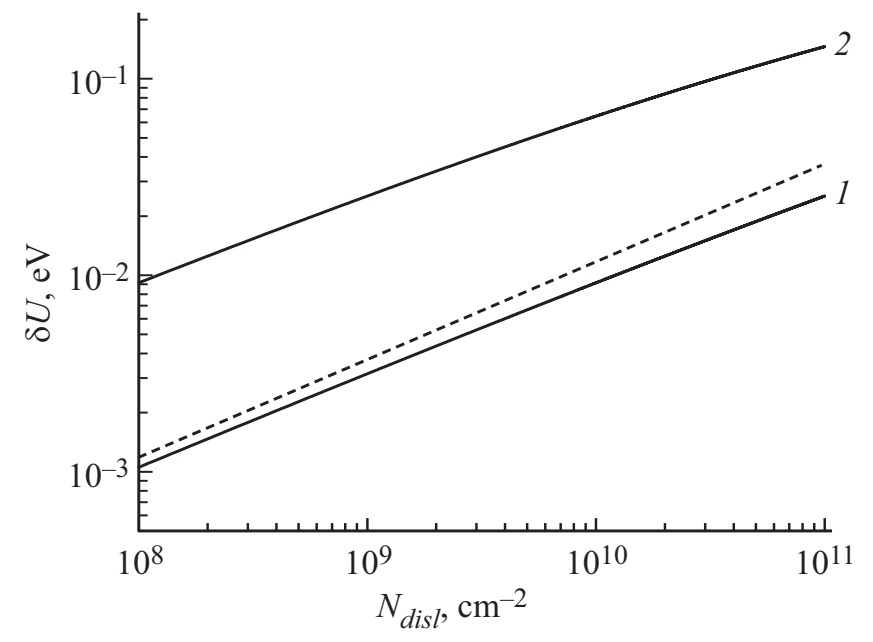

Зависимость амплитуды хаотического потенциала заряженных дислокаций ( $\lambda=0.01 \mathrm{CGS}$ units) в гетероконтакте $\mathrm{AlGaN} / \mathrm{GaN}$ от их концентрации при значениях плотности поверхностных состояний $D_{s}=10^{14}$ (1) и $10^{13} \mathrm{~cm}^{-2} \cdot \mathrm{eV}^{-1}$ (2). Штриховой линией показан результат расчета в приближении Томаса-Ферми (формула (6)) при $D_{s}=10^{14} \mathrm{~cm}^{-2} \cdot \mathrm{eV}^{-1}$.

Из определения данной функции [10] следует, что $\Phi(0)=1$ и ее асимптотическое поведение $\Phi(y) \sim 1 / y$ при условии $y \gg 1$. Типичная зависимость величины $\delta U$ от концентрации заряженных дислокаций $N_{d i s l}$ при двух значениях плотности поверхностных состояний приведена на рисунке.

Подведем некоторые итоги проведенного анализа. Расчет показывает (см. рисунок), что при практически максимальном заполнении акцепторных дислокационных состояний (0.01 CGS units соответствует плотности около 1 электрона на постоянную решетки [2]) наличие исходно в рассматриваемой системе сильно вырожденного делокализованного двумерного электронного газа высокой плотности (порядка $10^{14} \mathrm{~cm}^{-2} \cdot \mathrm{eV}^{-1}$ и более) приводит лишь к незначительным средним флуктуациям потенциальной энергии электрона, не превышающим тепловой энергии при комнатной температуре. При этом характерный масштаб данных флуктуаций в приведенном диапазоне концентраций линейных дефектов, согласно формуле (11), составляет от сотен до десятков ангстрем, что меньше среднего расстояния между ближайшими дислокациями. Но при уменьшении плотности поверхностных состояний на порядок увеличиваются в несколько раз как величины $\delta U$, так и значения $R_{0}$. При $N_{d i s l}=2 \cdot 10^{10} \mathrm{~cm}^{-2}$ величина $\delta U$ уже оказывается порядка $100 \mathrm{meV}$ и значение $R_{0}$ сравнимо с величиной $N_{d i s l}^{-1 / 2}$. Другими словами, в этих условиях хаотический потенциал перестает быть мелкомасштабным и необходимо учитывать частичную локализацию состояний $[11,12]$. Следует также отметить, что приближение Томаса-Ферми дает для амплитуды хаотического потенциала несколько завышенный результат.

\section{Финансирование работы}

Работа выполнена при поддержке Российского фонда фундаментальных исследований (проект № 19-52-80019 БРИКС_Т).

\section{Конфликт интересов}

Авторы заявляют, что у них нет конфликта интересов.

\section{Список литературы}

[1] N.G. Weimann, L.F. Eastman, D. Doppalapudi, H.M. Ng, T.D. Maustakus, J. Appl. Phys., 83 (7), 3656 (1998).

[2] J. Debdeep, A.C. Gossard, U.K. Mishra, Appl. Phys. Lett., 76 (13), 1707 (2000).

[3] Д.Ю. Протасов, Т.В. Малин, А.В. Тихонов, А.Ф. Цацульников, К.С. Журавлев, ФТП, 47 (1), 36 (2013).

[4] В.Б. Бондаренко, М.В. Кузьмин, В.В. Кораблев, ФТП, 35 (8), 964 (2001).

[5] В.Ф. Гантмахер, Электроны в неупорядоченных средах (Физматлит, М., 2003).

[6] Л.Д. Ландау, Е.М. Лифшиц, Теоретическая физика. Электродинамика сплошных сред (Наука, М., 1982).

[7] В.Б. Бондаренко, С.Н. Давыдов, А.В. Филимонов, ФТП, 44 (1), 44 (2010).

[8] Т. Андо, А. Фаулер, Ф. Стерн, Электронные свойства двумерных систем (Мир, М., 1985).

[9] V.W.L. Chin, T.L. Tansley, T. Osotchan, J. Appl. Phys., 75 (11), 7365 (1994).

[10] И.С. Градштейн, И.М. Рыжик, Таблицы интегралов, сумм, рядов и произведений (Наука, М., 1971).

[11] Б.И. Шкловский, А.Л. Эфрос, Электронные свойства легированных полупроводников (Наука, М., 1979).

[12] В.Б. Бондаренко, А.В. Филимонов, ФТП, 51 (10), 1372 (2017). 